\title{
BRITE-Constellation: Input Catalogue
}

\author{
A. Kaiser ${ }^{1}$, K. Zwintz ${ }^{1}$, W.W. Weiss ${ }^{1}$ \\ ${ }^{1}$ Institut für Astronomie, Türkenschanzstrasse 17, 1180 Vienna, Austria
}

\section{Abstract}

BRITE (BRIght Target Explorer) is a satellite mission that will survey the sky, measuring the brightness and temperature variations of the brightest stars. In order to compile a mission target catalogue fundamental information of these stars was retrieved and a Hertzsprung-Russell (HR) was diagram compiled. Furthermore the feasibility of the targets was investigated by checking the surroundings for nearby bright contaminating stars.

\section{Introduction}

The BRITE-Constellation satellite mission (Moffat et al., 2006) will measure the brightness and temperature variations on timescales ranging from hours to months for the 534 brightest stars in the sky. Amongst others, BRITEConstellation will investigate the role stellar winds play in setting up future stellar life cycles, and reveal pulsations that will allow astronomers to probe luminous star histories and ages through asteroseismology.

\section{Target Inventory}

The Simbad astronomical database (http://simbad.u-strasbg.fr/) was searched for objects brighter than $\mathrm{V}=4 \mathrm{mag}$ and coordinates (RA, DE), color information (UBVRI, uvby $\mathrm{H} \beta, 2 \mathrm{MASS}$ ), object types, spectral types and parallaxes were retrieved for a total number of 534 stars. Using the VISAT database (http://ams.astro.univie.ac.at/visat) a cross identification for star types was done. The determination of the fundamental parameters ( $T_{\text {eff }}, \log g, F e / H$, mass, radius, ...) was done with the TempLogG TNG software (http://ams.astro.univie.ac.at). The absolute magnitude $M_{\mathrm{v}}$ was derived from Hipparcos astrometric satellite parallaxes. 


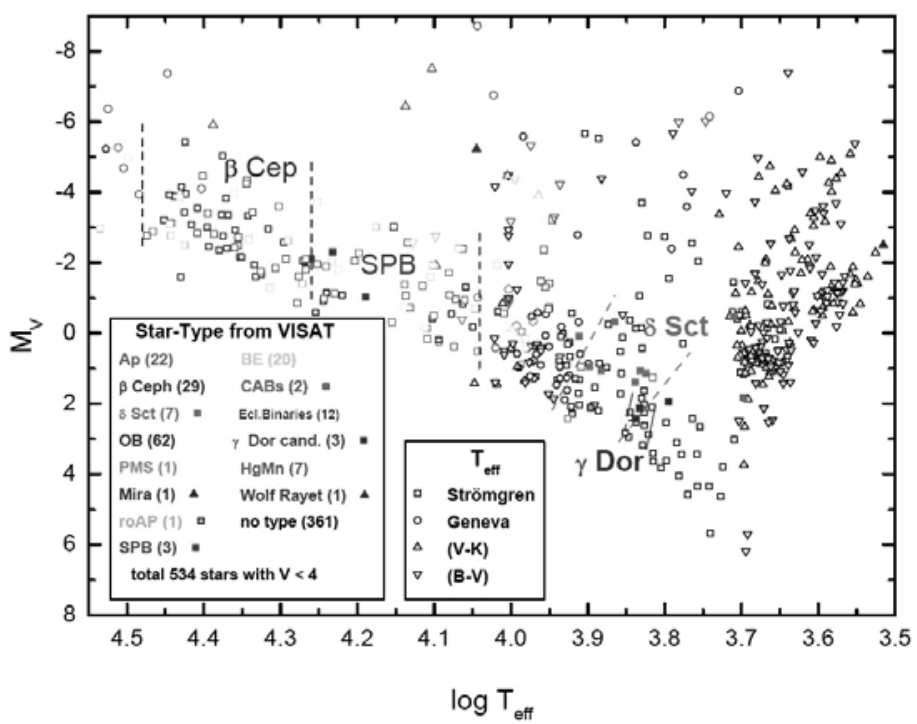

Figure 1: HR diagram of the 534 BRITE target stars with $V<4^{m}$. Temperatures have been derived from various color systems, while $M_{\mathrm{v}}$ was derived from Hipparcos astrometric satellite parallaxes.

\section{Target Contamination}

As the science instrument of BRITE will produce a quite defocused image of the target stars the surroundings of the potential BRITE targets were checked for close-by bright stars in order to assess the existence of enough feasible (i.e. uncontaminated) targets are existing. Background objects down to an apparent brightness of 15 mag were considered. The additional background flux was then computed with the assumption of a Gaussian like PSF of different full width at half maximum (FWHM). Figure 2 shows the additional flux in percent of the flux of the target star introduced by background objects for a FWHM of 5, 6 and pixels.

It shows that between 190 and 260 stars out of the total sample have contaminations lower than $0.2 \%$ and therefore are ideal main target candidates. Figure 3 and Figure 4 show the distribution of star types extracted from VISAT and Simbad for all targets with an additional flux of less than $0.2 \%$. 


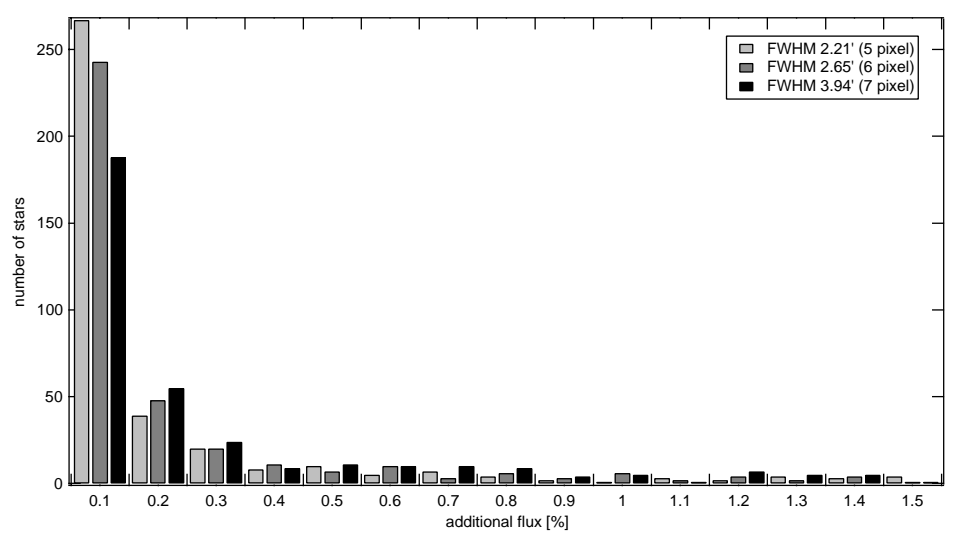

Figure 2: Additional flux in percent of the target star flux introduced by background objects.

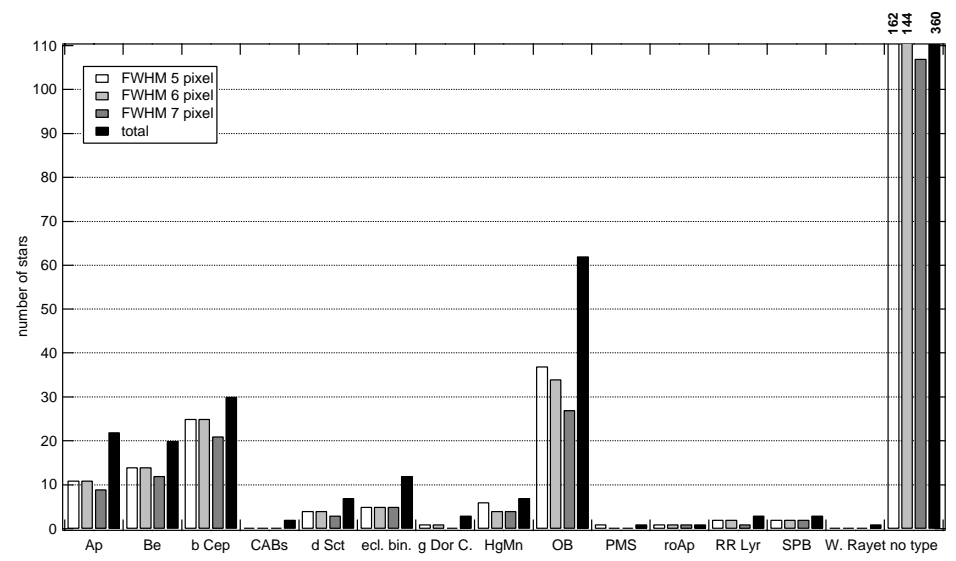

Figure 3: Stars with less than $0.2 \%$ additional flux with according star types from the VISAT database.

\section{Catalog Extension}

Extended simulations of the science instrument showed that a limiting magnitude of 4 is quite a conservative assumption. Therefore an extension of the input catalog including all stars with $4^{m} \leq V<6^{m}$ has been proposed by the BRITE Science Team (Figure 5). 


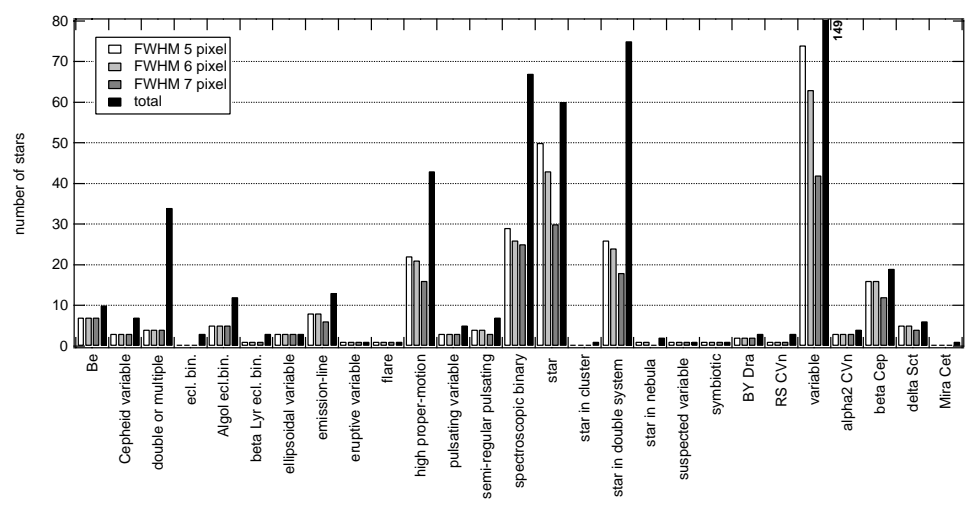

Figure 4: Stars with less than $0.2 \%$ additional flux with according star types from the SIMBAD database.

\section{Conclusions}

The BRITE-Constellation input catalogue consisting of 534 stars brighter than $\mathrm{V}=4 \mathrm{mag}$ has been compiled. Simulations have been performed in order to assess the optimal amount of defocusing of the camera system. Based on these simulations it was decided to aim for a PSF of 6 pixels FWHM. 


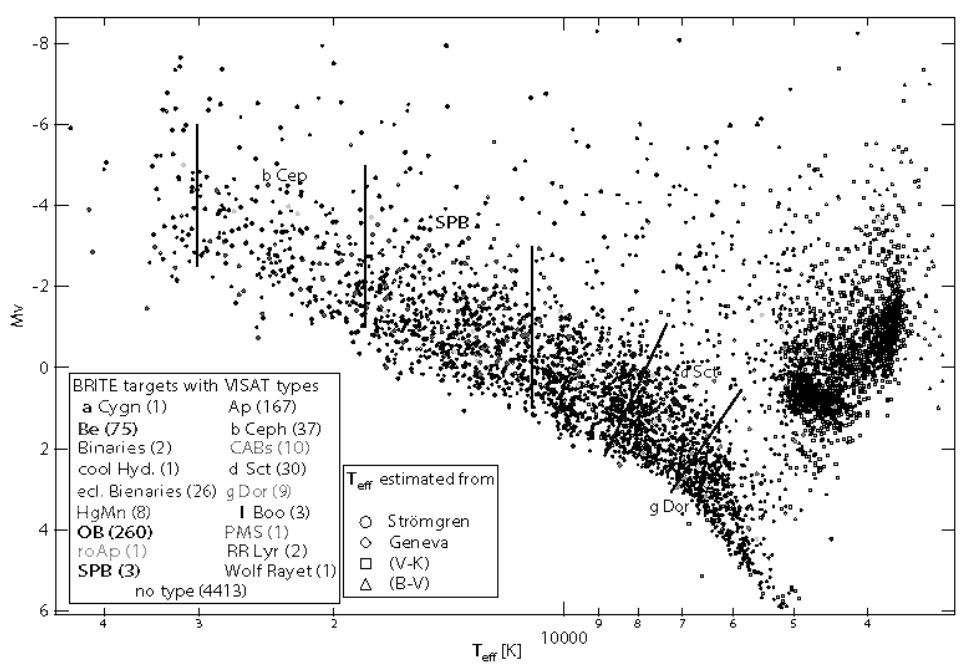

Figure 5: Same as Figure 1 but including the 4935 stars from the BRITE input catalog extension with $4^{m} \leq V<6^{m}$.

Acknowledgments. This research made use of the SIMBAD database, operated at CDS, Strasbourg, France and the VISAT database, operated by the SAPS research group at the Department of Astronomy, University Vienna. This research is supported by the Austrian Science Promotion Agency (BRITEAustria) and the University of Vienna (Uni-BRITE). Konstanze Zwintz acknowledges funding trough the Austrian Science Funds (FWF) project T335-N16.

\section{References}

Kaiser, A. 2006, ASPC, 349, 257

Kaiser, A., Weiss, W.W. 2007, CoAst, in preparation

Moffat, A.F.J., Weiss, W.W., Rucinski, S.M. et al. 2006, ASTRO $2006-13^{\text {th }}$ CASI Canadian Astronautics Conference 



\section{Science}


